\title{
Regulation of chromosome stability by the histone H2A variant Htz1, the Swr1 chromatin remodeling complex, and the histone acetyltransferase NuA4
}

\author{
Nevan J. Krogan ${ }^{\star \ddagger}$, Kristin Baetz ${ }^{\ddagger \S}$, Michael-Christopher Keogh ${ }^{\ddagger \rrbracket}$, Nira Datta*†, Chika Sawa", Trevor C. Y. Kwok ${ }^{\S}$, \\ Natalie J. Thompson*, Michael G. Davey*, Jeff Pootoolal*, Timothy R. Hughes* ${ }^{\dagger}$, Andrew Emili*, Stephen Buratowski", \\ Philip Hieter ${ }^{\S}$ and Jack F. Greenblatt**\|
}

\begin{abstract}
*Banting and Best Department of Medical Research and 'Department of Molecular and Medical Genetics, University of Toronto, Toronto, ON, Canada M5G 1L6; §Centre for Molecular Medicine and Therapeutics, Department of Medical Genetics, University of British Columbia, Vancouver, BC, Canada V5Z 4H4; and "Department of Biological Chemistry and Molecular Pharmacology, Harvard Medical School, Boston, MA 02115
\end{abstract}

Communicated by Robert G. Roeder, The Rockefeller University, New York, NY, August 5, 2004 (received for review May 10, 2004)

NuA4, the only essential histone acetyltransferase complex in Saccharomyces cerevisiae, acetylates the N-terminal tails of histones $\mathrm{H} 4$ and H2A. Affinity purification of NuA4 revealed the presence of three previously undescribed subunits, Vid21/Eaf1/ Ydr359c, Swc4/Eaf2/Ygr002c, and Eaf7/Ynl136w. Experimental analyses revealed at least two functionally distinct sets of polypeptides in NuA4: (i) Vid21 and Yng2, and (ii) Eaf5 and Eaf7. Vid21 and Yng2 are required for bulk histone $\mathrm{H} 4$ acetylation and are functionally linked to the histone H2A variant Htz1 and the Swr1 ATPase complex (SWR-C) that assembles Htz1 into chromatin, whereas Eaf5 and Eaf7 have a different, as yet undefined, role. Mutations in Htz1, the SWR-C, and NuA4 cause defects in chromosome segregation that are consistent with genetic interactions we have observed between the genes encoding these proteins and genes encoding kinetochore components. Because SWR-C-dependent recruitment of $\mathrm{Htz} 1$ occurs in both transcribed and centromeric regions, a NuA4/SWR-C/Htz1 pathway may regulate both transcription and centromere function in S. cerevisiae.

$T^{T}$ he nucleosomes that package eukaryotic DNA into chromatin can prevent the site-specific binding of many proteins to DNA. Two classes of factors have been implicated in regulating access to DNA in this context. The ATP-dependent chromatin-remodeling enzymes use energy derived from ATP hydrolysis to induce nucleosome mobility or disrupt histone-DNA interactions (reviewed in ref. 1). The second class of enzymes catalyzes covalent modification (e.g., lysine acetylation, serine phosphorylation, lysine and arginine methylation, ubiquitylation, and ADP ribosylation) of various histones, usually on their $\mathrm{N}$-terminal tails (reviewed in refs. 2 and 3). Nucleosome variability is also generated by occasional replacement of a major histone with a variant that has a specialized role (reviewed in ref. 4). Among H2A variants is H2A.Z (Htz1 in Saccharomyces cerevisiae), whose insertion into chromatin requires the recently described SWR1 complex (SWR-C) (5-7). H2A.Z is functionally distinct from the major histone H2A. Moreover, the various eukaryotic H2A.Z molecules are more closely related to each other than they are to H2A. This similarity in sequence extends to functional conservation, as H2A.Z from the ciliated protozoan Tetrahymena thermophila can rescue all tested phenotypes associated with S. cerevisiae htz1s (8). Htz1 plays a role in transcription $(5-7,9,10)$ and may act as a barrier to inhibit the spread of silent telomeric and mating locus hetereochromatin into adjacent transcriptionally active regions (9).

Histone acetylation is carried out by a class of enzymes known as histone acetyltransferases (HATs). These transfer an acetyl group from acetyl-CoA to lysine $\varepsilon$-amino groups on target histone $\mathrm{N}$ terminal tails. Histone acetylation has been linked to transcriptional activation, because actively transcribed regions are characteristically hyperacetylated, whereas silent regions are typically hypoacetylated (reviewed in ref. 11). The acetylation of lysine residues on the N-terminal tails of histones $\mathrm{H} 3$ and $\mathrm{H} 4$ neutralizes their positive charge, possibly decreasing their affinity for DNA and facilitating chromatin decompaction and disassembly. More important than simple charge neutralization, however, is the specific acetylation pattern at individual lysine residues within the histone $\mathrm{N}$ termini $(11,12)$. At least some acetylated lysines interact with and recruit bromodomain-containing polypeptides (ref. 13 and references therein). The HAT complexes of S. cerevisiae, many of which contain associated bromodomains, include the Gen5-containing ADA and SAGA complexes, Hat1, Elongator, NuA3, and NuA4. These complexes generally have high specificity for specific lysine residues on specific histone $\mathrm{N}$-terminal tails.

The catalytic subunit of NuA4, Esa1, is a member of the MYST (for MOZ, Ybf2/Sas3, Sas2, and Tip60) family of HATs (reviewed in ref. 14). In $S$. cerevisiae, NuA4 is required for minor acetylation on histone $\mathrm{H} 2 \mathrm{~A}$ and the majority of histone $\mathrm{H} 4$ acetylation on lysines 5,8 , and $12(15,16)$. Nine other NuA4 subunits have been reported: Esa1 associated factor 3 (Eaf3), a chromodomaincontaining factor also found in a Sin $3 / \mathrm{Rpd} 3$ histone deacetylase complex (17); Eaf5 and Eaf6; Tra1, a member of the phosphatidylinositol kinase family; the actin-related proteins Act1 and Arp4; Yng2, an ING1 tumor suppressor homolog; Epl1, a homolog of the Enhancer of Polycomb-like protein; and Yaf9, a protein related to the AF9/ENL leukemogenic factors.

Here, we report the identification of three NuA4 subunits, namely Vid21/Eaf1/Ydr359c, Swc4/Eaf2/Ygr002c, and Eaf7/ Ynl136w. Vid21 and Swc4 both contain SANT (WWI3, ADA2, N-CoR, TFIIIB) domains, also found in several other chromatin remodeling complexes. Swc4, Yaf9, Arp4, and Act1 are also components of the SWR-C (5-7). Microarray and genetic analyses revealed at least two functionally distinct classes of polypeptides in NuA4. The first class contains Vid21 and Yng2, required for most of the acetylation activity of Esa1. The second class, containing Eaf5 and Eaf7, plays a minor role, if any, in acetylation of histone H4 by NuA4.

We showed previously that the SWR-C recruits Htz1 to various regions of the genome (5), and we show here that this is also the case at the centromere. Although NuA4 is not required for assembling Htz1 into chromatin, Htz1 and subunits of NuA4 and the SWR-C are genetically linked to kinetochore components and are important for chromosome stability. Genetic analyses suggest that these factors operate in common, rather than parallel, pathways. These observations imply that NuA4, the SWR-C, and Htz1 function

Abbreviations: SWR-C, SWR1 complex; HAT, histone acetyltransferase; Eaf, Esa1-associated factor; TAP, tandem affinity purification; MALDI-TOF, matrix-assisted laser desorption ionization time-of-flight; SGA, synthetic genetic array; MS/MS, tandem MS; ChIP, chromatin immunoprecipitation.

₹N.J.K., K.B., and M.-C.K. contributed equally to this work.

\|To whom correspondence should be addressed. E-mail: jack.greenblatt@utoronto.ca.

(c) 2004 by The National Academy of Sciences of the USA 
A

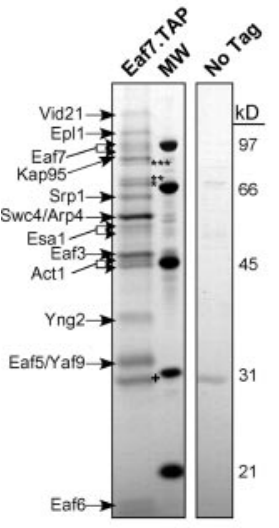

B

\begin{tabular}{|c|c|c|}
\hline Vid21 & Eaf1 & YDR359C \\
\hline Epl1 & - & YFL024C \\
\hline Swc4 & Eaf2 & YGR002C \\
\hline Arp4 & - & YJL081C \\
\hline Esa1 & - & YOR244W \\
\hline Eaf7 & - & YNL136W \\
\hline Eaf3 & - & YPR023W \\
\hline Act1 & - & YFL039C \\
\hline Yng2 & Eaf4 & YHR090C \\
\hline Eaf5 & - & YEL018W \\
\hline Yaf9 & - & YNL107W \\
\hline Eaf6 & - & YJR082C \\
\hline Tra1 & & YHR099W \\
\hline Bdf1 & & YLR399C \\
\hline Hmo1 & & YDR174W \\
\hline H2A & & YDR225W \\
\hline Htz1 & & YOL0012C \\
\hline
\end{tabular}

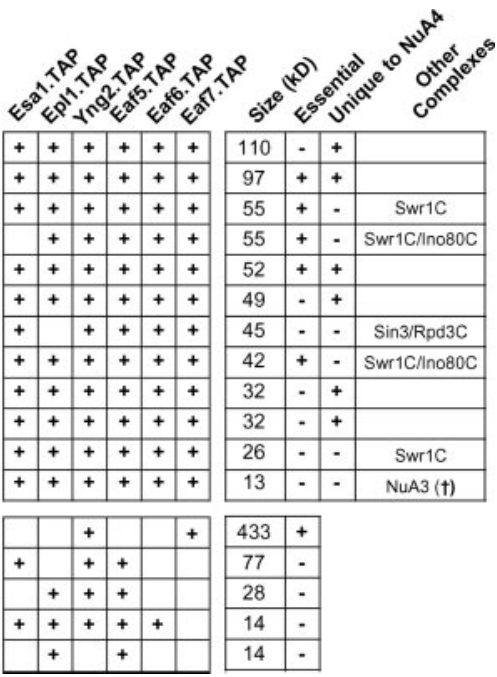

Fig. 1. Isolation of the NuA4 HAT complex. $(A)$ TAP of the NuA4 complex was carried out on strains containing either TAP-tagged Eaf7 or no tagged protein. The purified protein was analyzed by SDS/PAGE and silver staining. The indicated proteins were identified by trypsin digestion of each stained band followed by MALDI-TOF mass spectrometry or tandem mass spectrometry after subjecting an aliquot of the eluate from the final column directly to trypsin. Contaminating bands are indicated: ***, Sse $1 ; * *$, Ssa1; *, Ssb1; †, TEV protease. The Srp1/Kap95 importin complex, which transports NLS-containing proteins into the nucleus (56), also copurified with NuA4 and is often found associated with nuclear protein complexes (N.J.K., A.E. and J.F.G., unpublished data). (B) Summary of purified proteins identified by mass spectrometry. Proteins that were present in at least two of the six purifications are represented. Protein size (kDa) was as predicted by amino acid composition (Saccharomyces Genome Database; www.yeastgenome.org) and not determined experimentally. (†, N.J.K., A.E., and J.F.G., unpublished data.) together to regulate chromosome stability and/or transmission as well as transcription in $S$. cerevisiae.

\section{Experimental Procedures}

Tandem affinity purification (TAP)-tagged components of NuA4 were purified on IgG and calmodulin columns from extracts of yeast cells (3 liters) grown in yeast extract/peptone/dextrose (YPD) medium to an $\mathrm{OD}_{600}$ of $1.0-1.5$. Complexes were analyzed by SDS/PAGE, matrix-assisted laser desorption ionization time-offlight (MALDI-TOF) MS, and tandem MS (MS/MS) as described (18). Synthetic genetic array (SGA) analysis was carried out as described (19). Automated analysis of the results was carried out by procedures that are described elsewhere $(\mathrm{H}$. Ding and C. Boone, personal communication). RNA preparation and microarray analysis were as described $(20,21)$ after isogenic strains were grown in parallel in $\mathrm{SC}$ medium at $30^{\circ} \mathrm{C}$. Chromatin immunoprecipitation (ChIP) assays using the TAP-tagged strains were performed essentially as described $(5,18)$. For ChIP analysis in the context of temperature-sensitive alleles of esa1 (esa1- $\Delta 414$, esa1-L327S; ref. 22), crosslinking was performed at $25^{\circ} \mathrm{C}$ or after $3 \mathrm{~h}$ at $37^{\circ} \mathrm{C}$ and compared to an isogenic wild-type strain under the same conditions. IgG agarose for precipitation of TAP-tagged proteins was from Sigma. All primer sequences are listed in Table 2, which is published as supporting information on the PNAS web site. Protein isolation and Western blot procedures were performed as described (23) with modifications described in Supporting Text, which is published as supporting information on the PNAS web site.

Quantitative half-sector analysis was performed as described (24-26). In brief, homozygous diploid strains (YPH982, wild-type; YKB522, eaf1s; YKB463, eaf7s; YPH493, yaf9s; YKB508, swr1s; YKB503, htz1 $\Delta$ ) (see Table 3, which is published as supporting information on the PNAS web site) were created containing an ade $2^{\text {ochre }}$ allele at the endogous locus and the SUP11 ochre suppressor on a single chromosome fragment [CFIII (CEN3. L) URA3 SUP11]. Strains were plated to single colonies on solid media containing limiting adenine and grown at $25^{\circ} \mathrm{C}$ for 3 days before the plates were placed at $4^{\circ} \mathrm{C}$ for optimal red pigment development. Efficient chromosome stability/transmission results in pink colonies. Chromosome loss or 1:0 events were scored as colonies that were half red and half pink, nondisjunction or 2:0 events were scored as colonies that were half red and half white.

\section{Results and Discussion}

To further characterize the subunit composition of yeast NuA4, we used single-step transformation to place a TAP tag (17) at the C terminus of several of its known components (Esa1, Epl1, Yng2,
Eaf5, and Eaf6). The tagged protein was then purified sequentially on IgG and calmodulin columns and analyzed by SDS/PAGE followed by staining with silver. Protein bands absent from a control preparation and corresponding to the tagged factor and any associated proteins were identified by MALDI-TOF MS and MS/MS.

In each case, the tagged protein copurified with all, or almost all, of the known subunits of the NuA4 complex (Fig. $1 B$ and data not shown). Only the $400-\mathrm{kDa}$ subunit Tra1 was not identified by gel band excision and MALDI-TOF MS, even though Tra1 is a well characterized stoichiometric component of the NuA4 complex (16) and silver-stained bands were present at this molecular weight in almost every purified preparation. Tra1 was, however, detected by using liquid chromatography (LC) MS/MS after the purification of Yng2-TAP (Fig. 1B Lower). In each purified preparation, we detected three previously unreported subunits of NuA4, namely Vid21/Eaf1/Ydr359c, Swc4/Eaf2/Ygr002c, and Eaf7/Ynl136w (Fig. $1 B$ ). The tagging and purification of Eaf7 confirmed that this subunit is indeed a member of the complex (Fig. 1). In this case, we again detected Tra1 as copurifying with the NuA4 complex. Eaf7TAP also copurified with Vid21 and Swc4, both of which contain SANT domains that are often present in complexes involved in chromatin metabolism. Vid21 copurified with each tagged subunit of NuA4 that we isolated (Fig. 1B). Interestingly, Swc4 is also a component of the SWR-C chromatin remodeling complex required for replacing histone $\mathrm{H} 2 \mathrm{~A}$ with the $\mathrm{H} 2 \mathrm{~A}$ variant, $\mathrm{Htz1}(5,6)$. Purification of tagged Swc4 led to the isolation of a mixture of NuA4 and the SWR-C (data not shown). Other shared components of the SWR-C and NuA4 include Arp4, Act1, and Yaf9 (Fig. 1B). The human Tip60 HAT complex contains factors homologous to those in both the SWR-C and NuA4 (27-29), consistent with the observation that components are shared between these complexes in S. cerevisiae.

In three of the six purified preparations, we detected the highmobility group (HMG) domain protein, Hmo1, by LC/MS/MS (Fig. $1 B)$. There are seven proteins in $S$. cerevisiae that contain a $\mathrm{HMG}$ DNA-binding domain, several of which, including Hmo1, bind to rDNA and are linked to RNA polymerase I transcription (30). An association between $\mathrm{NuA} 4$ and $\mathrm{Hmo} 1$ may partly explain the observed role of NuA4 in rDNA transcription (31). NuA4 also copurified with small amounts of histone $\mathrm{H} 2 \mathrm{~A}$, a known substrate of $\mathrm{NuA} 4$, as well as with small amounts of Bdf1 and the histone $\mathrm{H} 2 \mathrm{~A}$ variant, Htz1, which may represent potential substrates. Bdf1 is also associated with the SWR-C (5) and binds to acetylated histone tails (13).

Genes encoding proteins in the same functional pathway should, in principle, have similar effects on gene expression and similar sets 
A

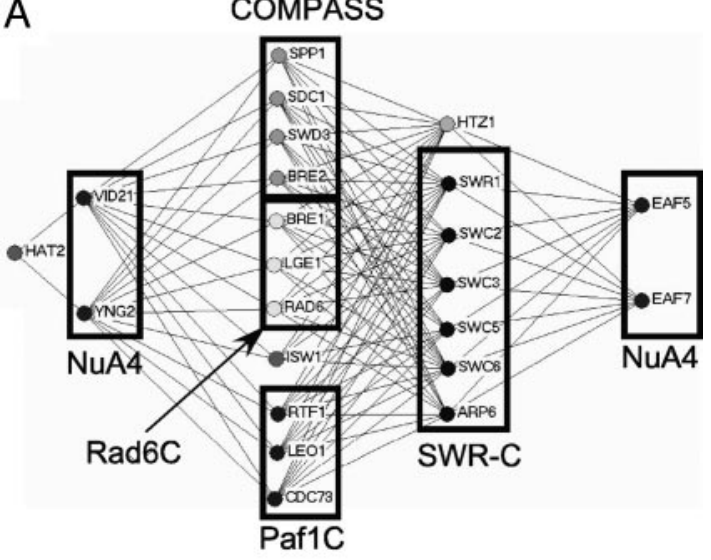

B

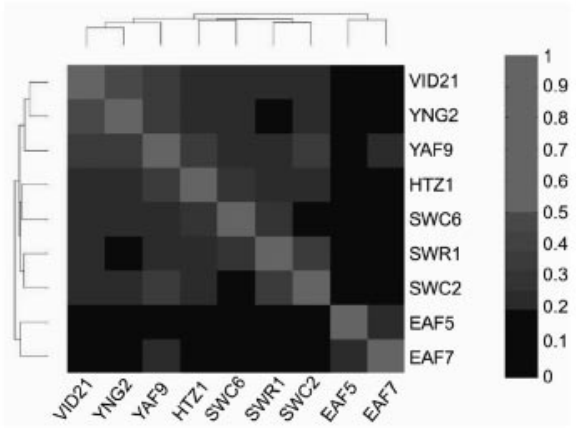

C

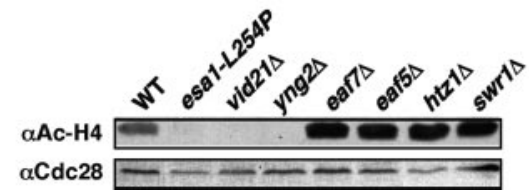

Fig. 2. Genetic similarity of the SWR-C and a subset of NuA4. (A) Synthetic genetic interactions for the SWR-C and NuA4. SGA technology (19) was used to cross $\mathrm{Nat}^{r}$ strains harboring individual deletions of genes encoding $\mathrm{Htz} 1$ or subunits of the SWR-C or NuA4 with a transcription-targeted array of $384 \mathrm{Kan}^{\mathrm{R}}$ deletion strains to create sets of $\mathrm{Nat}^{r} \mathrm{Kan}^{r}$ haploid double mutants. Growth rates were assessed by automated image analysis of colony size. Lines connect genes with synthetic genetic interactions. The lengths of lines and proximity of boxes in this diagram and in Fig. $3 A$ are unrelated to the strengths of the indicated synthetic genetic interactions. $(B)$ Microarray analysis of gene expression was performed for the indicated deletion strains. Pearson correlation coefficients were then calculated for each pair of deletions, and the deletions were organized by 2D hierarchical clustering according to the similarities of their effects. (C) Immunoblots of whole-cell protein extracts from wild-type

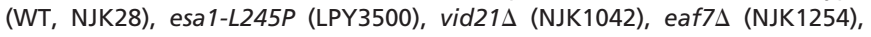

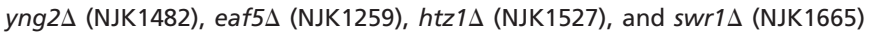
cells (see Table 3 ) grown to log phase in yeast extract/peptone/dextrose at $30^{\circ} \mathrm{C}$ and transferred to $37^{\circ} \mathrm{C}$ for $4 \mathrm{~h}$. The membranes were probed with antihyperacetylated histone $\mathrm{H} 4$ (Penta) antibodies ( $\alpha \mathrm{Ac}-\mathrm{H} 4)$ and then reprobed with antibodies to $\mathrm{Cdc} 28$ to verify approximately equal protein loading.

of synthetic genetic interactions. To test this concept and provide independent evidence that the identified proteins unique to $\mathrm{NuA} 4$ are functionally significant components of the complex, we first used automated SGA analysis (19). For this purpose, a miniarray containing $384 \mathrm{Kan}^{\mathrm{r}}$ deletion strains was constructed in which each deletion represents a protein known or suspected to function in some aspect of transcription and/or chromatin modification or remodeling (N.J.K. and J.F.G., unpublished data). Four Nat ${ }^{\mathrm{R}}$ strains harboring individual gene deletions of nonessential components of NuA4 (VID21, YNG2,EAF5, and EAF7) were then generated and crossed with the 384 deletion strain miniarray to create sets of $\operatorname{Kan}^{\mathrm{r}} \mathrm{Nat}^{\mathrm{r}}$ haploid double mutants. Genetic interac-

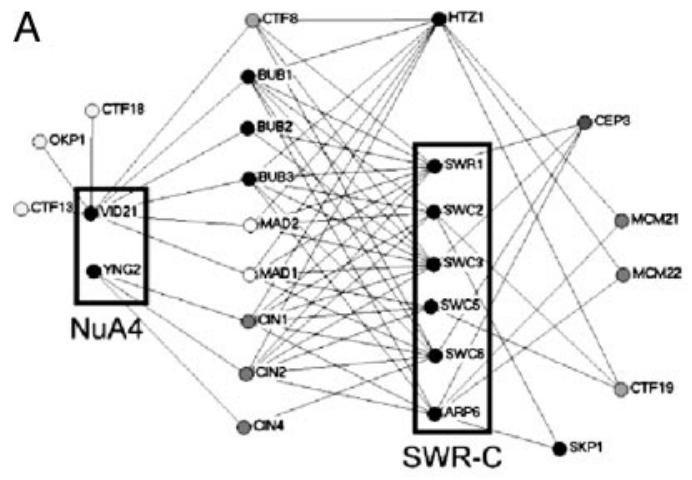

B

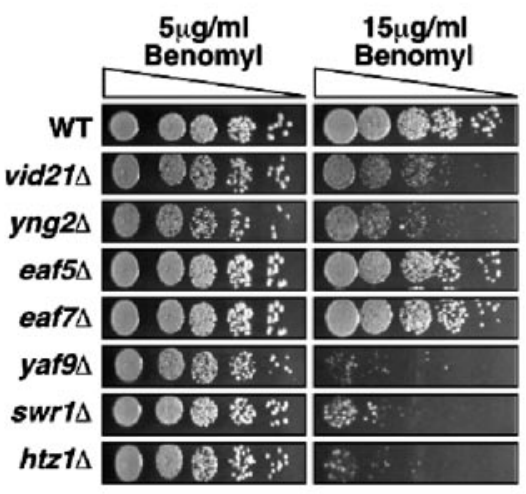

Fig. 3. Htz1, SWR-C, and the NuA4 complex function to regulate chromosome stability/transmission. (A) SGA analysis (19) using either a transcriptiontargeted 384-deletion-strain array or genome-wide kinetochore screens (K.B. and V. Measday, unpublished data) identified numerous synthetic genetic interactions between deletions of genes encoding Htz1, SWR-C, or the NuA4 subunits Vid21 and Yng2, and known chromosome stability/transmission factors (see text for details). Genome-wide screens were carried out with four essential kinetochore genes [skp1-3 (42), cep3-1 (43), ctf13-30 (44), and okp1-5 (45)], whereas the nonessential components ( $m c m 21 \Delta, \mathrm{mcm} 22 \Delta$, $c t f 19 \Delta$, bub1 $\Delta$, bub3 $\Delta, \operatorname{mad} 1 \Delta$, and $\operatorname{mad} 2 \Delta$ ) were present on the targeted miniarray. $(B)$ Effects of the microtubule destabilizing agent benomyl on the growth of wild-type (NJK28), vid21 $($ NJK1042), eaf5 $\Delta$ (NJK1259), eaf7 $\Delta$

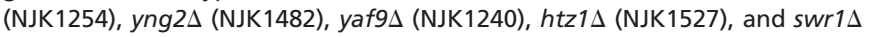
(NJK1665) strains. Five-fold serial dilutions of strains starting from an $\mathrm{OD}_{600}$ of 0.1 were plated onto yeast extract/peptone/dextrose plates containing 5 or $15 \mu \mathrm{g} / \mathrm{ml}$ benomyl and incubated for 2 days at $30^{\circ} \mathrm{C}$.

tions identified for vid21 $\Delta$ and $y n g 2 \Delta$ were very similar but dramatically different from those observed with eaf5 $\Delta$ and eaf $7 \Delta$, which, in turn, were very similar to each other (Fig. $2 A$ and data not shown). Strains harboring a deletion of EAF5 or EAF7 were synthetically sick when combined with a deletion of $H T Z 1$ or genes encoding nonessential components of the SWR-C. This finding suggests that these subunits of $\mathrm{NuA} 4$ act in a distinct pathway, possibly parallel to that of the SWR-C and Htz1. In contrast, vid21 $\Delta$ and $y n g 2 \Delta$ lacked these interactions, and instead interacted genetically with different genes encoding factors required for histone $\mathrm{H} 3$ $\mathrm{K} 4$ methylation, as was also seen with $h t z 1 \Delta$ and deletion of genes encoding components of the SWR-C (Fig. 2A). This observation included components of the Set1 histone methyltransferasecontaining complex, COMPASS (SPP1, SDC1, SWD3, and BRE2) (32); the PAF complex (RTF1, LEO1, and CDC73), which is required for the recruitment of COMPASS to chromatin $(33,34)$; and the Rad6 complex (RAD6, BRE1, and LGE1) that ubiquitinates $\mathrm{K} 123$ of histone $\mathrm{H} 2 \mathrm{~B}$, an event required for methylation of $\mathrm{H} 3$ K4 by COMPASS $(35,36)$. Finally, genetic interactions with the Snf2-family member and chromatin remodeler $I S W 1$, the function of which has been linked to histone H3 K4 methylation (37), were also observed. The similar patterns of genetic interactions for the 
Table 1. Rates of chromosome missegregation events

\begin{tabular}{|c|c|c|c|}
\hline \multirow[b]{2}{*}{ Genotype } & \multicolumn{2}{|c|}{ Rate } & \multirow[b]{2}{*}{$\begin{array}{c}\text { Total } \\
\text { colonies }\end{array}$} \\
\hline & $\begin{array}{l}\text { Nondisjunction } \\
\text { (2:0 events) }\end{array}$ & $\begin{array}{l}\text { Chromosome } \\
\text { loss (1:0 events) }\end{array}$ & \\
\hline Wild type* & $8.7 \times 10^{-5}(1.0)$ & $8.7 \times 10^{-5}(1.0)$ & 22,800 \\
\hline $\operatorname{vid} 21 \Delta$ & $9.5 \times 10^{-4}(10.9)$ & $3.6 \times 10^{-3}(41.5)$ & 16,875 \\
\hline eaf7 $\Delta$ & $6.7 \times 10^{-4}(7.7)$ & $1.2 \times 10^{-3}(13.8)$ & 29,670 \\
\hline yafgs & $1.7 \times 10^{-4}(2.0)$ & $2.0 \times 10^{-3}(23.0)$ & 17,400 \\
\hline swr1s & $3.0 \times 10^{-4}(3.4)$ & $6.2 \times 10^{-4}(7.2)$ & 30,450 \\
\hline$h t z 1 \Delta$ & $1.2 \times 10^{-4}(1.4)$ & $1.3 \times 10^{-3}(14.5)$ & 32,585 \\
\hline
\end{tabular}

Strains were plated to single colonies, and sectoring colonies were scored visually as described in Experimental Procedures. Colonies scored as halfsectored were $\geq 50 \%$ red (24). Strains used were YPH982, YKB522, YKB46, YPH493, YKB508, YKB513, and YKB503. Numbers in parentheses are the factors of increase in rate of missegregation events above wild type. The rates observed are comparable to those described after mutation/deletion of characterized chromosome stability/transmission factors.

*Wild-type rates were published in ref. 26.

genes encoding the NuA4 subunits Vid21 and Yng2, Htz1, and members of the SWR-C suggested that these proteins function in a common pathway in vivo.

We have also individually crossed $\mathrm{Nat}^{\mathrm{R}}$ versions of each of the 384 deletion strains in our transcription/chromatin modification miniarray against the entire $\mathrm{Kan}^{\mathrm{R}}$ miniarray, thereby creating a $384 \times 384$ matrix of double mutant strains (N.J.K. and J.F.G., unpublished data). Growth rates were evaluated by automated image analysis, and the results of all of the screens were analyzed by $2 \mathrm{D}$ hierarchical clustering. This clustering process groups genes according to the degrees of similarity of their synthetic genetic interactions. One important expectation is that genes with the same or very similar functions would have similar sets of genetic interactions and would fall into the same cluster. The genetic patterns obtained from strains containing deletions of either VID21 or $Y N G 2$ were more similar to each other than to those obtained from any of the other 382 genes on the array (i.e., these genes clustered next to each other), implying that they have a very similar role in NuA4 function (data not shown). EAF5 and EAF7 also clustered next to each other, but away from VID21 and YNG2. This finding indicated that Eaf5 and Eaf7 are functionally similar subunits of $\mathrm{NuA4}$, but that their roles are distinct from those of Vid21 and Yng2. Interestingly, HTZ1 and genes encoding components of the SWR-C clustered near VID21 and YNG2, but away from EAF5 and EAF7 (data not shown), again suggesting that only certain components of NuA4 function with the SWR-C in vivo.

To further characterize these seemingly functionally distinct sets of proteins contained within $\mathrm{NuA} 4$, we carried out microarray analyses of gene expression in strains containing deletions of $V I D 21, Y N G 2, E A F 5$, and $E A F 7$. For comparison purposes, we also conducted microarray experiments on strains deleted for HTZ1 and components of the SWR-C (SWR1, SWC2, and SWC6, as well as YAF9, a subunit of both NuA4 and SWR-C) (raw microarray data are available at www.utoronto.ca/greenblattlab/ nua4swrc.xls). Pearson correlation coefficients were calculated for each pair of deletions, and the strains were organized by $2 \mathrm{D}$ hierarchical clustering according to the similarities of their effects on gene expression (Fig. 2B).

As was observed with our genetic analyses, the gene expression profiles of strains containing deletions of VID21 and YNG2 were similar to each other and to those of strains containing deletions of HTZ1 or components of the SWR-C. Deletion of YAF9, which encodes a shared component of the SWR-C and NuA4, had a very similar effect on gene expression (Fig. $2 B$ ), consistent with a functional role in both complexes. In contrast, EAF5 and EAF7 clustered next to each other but away from the other genes in this gene expression analysis, just as they had in our genetic analysis.
Microarray experiments have also been conducted on nearly 200 other deletion strains represented on our 384-strain genetic miniarray (N.J.K., T.R.H., and J.F.G., unpublished data). The clustering of this larger data set (not shown) reflects the data represented in Fig. $2 B$, in which VID21 and YNG2 clustered next to HTZ1 and genes encoding components of the SWR-C and away from EAF5 and $E A F 7$. Therefore, both the genetic and gene expression data suggest that the Vid21 and Yng2 subunits of NuA4 function in an SWR-C/Htz1 pathway, whereas Eaf5 and Eaf7 are important for a different, perhaps parallel, role of NuA4.

To identify another functional distinction among these NuA4 subunits, we took advantage of the fact that $\mathrm{NuA} 4$ is responsible for most histone $\mathrm{H} 4$ acetylation in $\mathrm{S}$. cerevisiae. Whole cell extracts were prepared from esa1-L254P, a temperature-sensitive allele with diminished HAT activity (22), or strains containing deletions of various NuA4 subunits and probed with antibody specific for hyperacetylated histone $\mathrm{H} 4$. Whereas deletion of EAF5 or EAF7 had no apparent effect on histone $\mathrm{H} 4$ acetylation, deletion of VID21 or YNG2 appeared to result in its complete loss (Fig. $2 C$ ). It has been reported that Yng2, but not Eaf5, is required for the catalytic activity of NuA4 (38). VID21 and YNG2, but not EAF5 or EAF7, interacted genetically with $H A T 2$, a component of the histone acetyltransferase B complex (39) (Fig. 2A), consistent with the fact that Vid21 and Yng2 are involved in H4 acetylation by NuA4. Acetylation elsewhere (or below the threshold of detection) by $\mathrm{NuA} 4$ in the absence of Vid21 or Yng2 must, however, exist, because the catalytic subunit, Esa1, is essential (15), whereas Vid21 and Yng2 are not. Further comparison with the genetic data summarized in Fig. $2 \mathrm{~A}$ indicated that $\mathrm{H} 4$ acetylation by $\mathrm{NuA} 4$ (or acetylation elsewhere that also depends on Vid21 and Yng2) and H3 K4 methylation by COMPASS cannot be eliminated simultaneously.

We recently identified VID21 in a ctf13-30/CTF13 genome-wide modifier screen to identify genes whose function impinges on chromosome transmission (26). Deletion strains of VID21 were shown to be sensitive to overexpression of the inner kinetochore genes $C T F 13$ and $C B F 2$ and display a high rate of chromosome loss (26). These observations suggested that $\mathrm{NuA} 4$ might be involved in centromere function as well as transcription. Our SGA screen has identified numerous synthetic genetic interactions between Htz1, the SWR-C, NuA4 (VID21 and YNG2), and known kinetochore and spindle checkpoint mutants (Fig. $3 A$ ). The kinetochore is a large multiprotein complex that binds centromeric DNA and serves to link chromosomes to spindle microtubules. The kinetochore both mediates and monitors its interaction with the spindle by communicating attachment defects to the checkpoint machinery (reviewed in refs. 40 and 41). In particular, we observed genetic interactions with mutation or deletion of subunits of the C-repeat binding factor 3 inner kinetochore complex [skp1-3 (42), cep3-1 (43), or ctf13-30 (44)] and subunits of the central kinetochore complexes [okp1-5 (45), $m c m 21 \Delta, m c m 22 \Delta$, or ctf19s]. We also detected genetic interactions with mutants of components of the spindle checkpoint, bub1 1 , bub3 3 , mad1 $\Delta$, mad2s (46), the spindle position checkpoint, $b u b 2 \Delta$ (47), and genes required for microtubule stability, $\operatorname{cin} 1 \Delta, \operatorname{cin} 2 \Delta$, and $\operatorname{cin} 4 \Delta(48)$. Together, these genetic interactions also suggested that Htz1, NuA4, and the SWR-C may be required for chromosome stability in addition to their previously described roles in transcription.

Numerous kinetochore and spindle checkpoint mutants are hypersensitive to the microtubule destabilizing drug benomyl. This is also the case for certain NuA4 mutants, including esa1-1851, yaf $9 \Delta$, yng $2 \Delta$, and vid $21 \Delta$ (refs. 7 and 23 and Fig. $3 B$ ). In contrast, eaf $5 \Delta$ and eaf $7 \Delta$ strains displayed no sensitivity to benomyl, an observation that is consistent with our microarray and genetic data pointing to different roles for these NuA4 subunits (Fig. 2). Interestingly, benomyl sensitivity is also observed after deletion of HTZ1 or the catalytic subunit of the SWR-C, SWR1 (Fig. $3 B$ and ref. 7). Although the NuA4-SWR-C-Htz1 pathway interacts genet- 
A
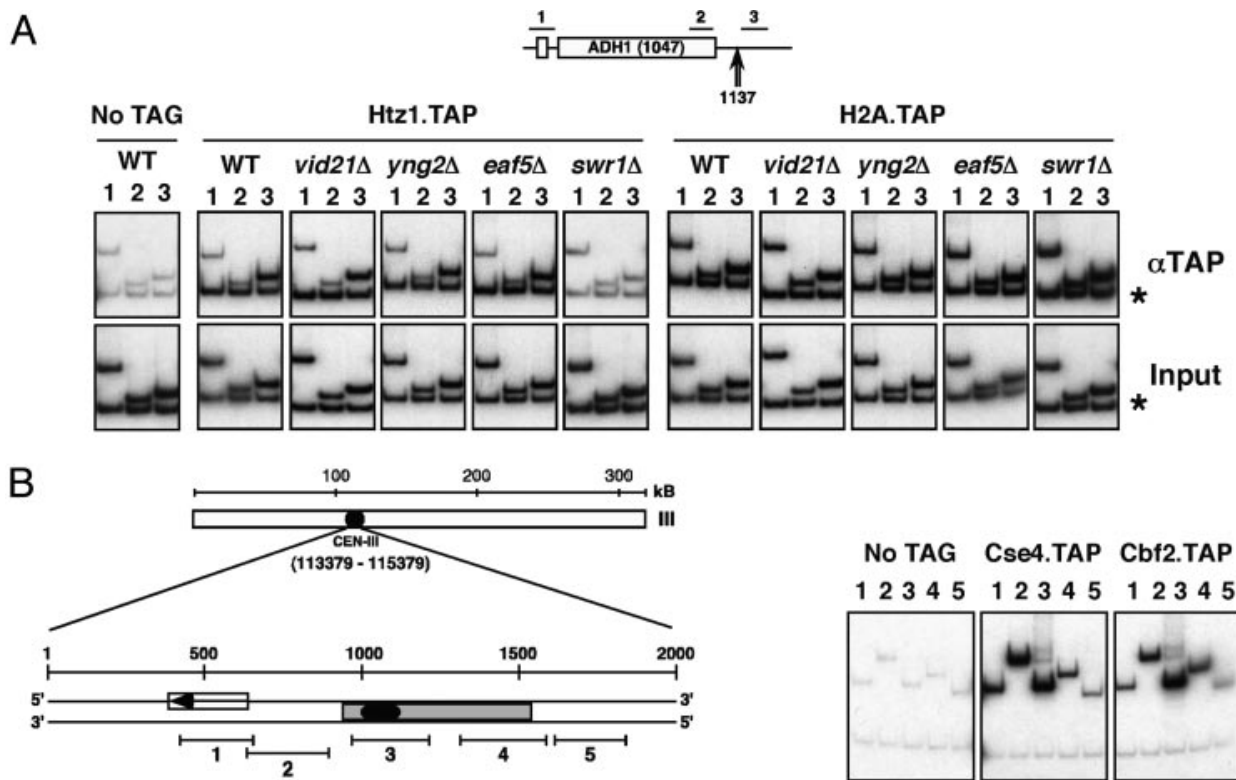

\begin{tabular}{c|c|c} 
ORF \# & $\begin{array}{c}\text { mRNA } \\
/ \mathrm{hr}\end{array}$ & Location \\
\hline YCL001W-B & 0.7 & $113764-114018$ \\
$\begin{array}{c}\text { ARS308 } \\
\text { CEN3 }\end{array}$ & - & $114315-114933$ \\
& - & $114379-114495$
\end{tabular}

\begin{tabular}{c|c}
$\begin{array}{c}\text { Primer } \\
\text { pair }\end{array}$ & Location \\
\hline 1 & $113807-114043$ \\
2 & $114019-114283$ \\
3 & $114314-114565$ \\
4 & $114656-114934$ \\
5 & $114966-115197$
\end{tabular}

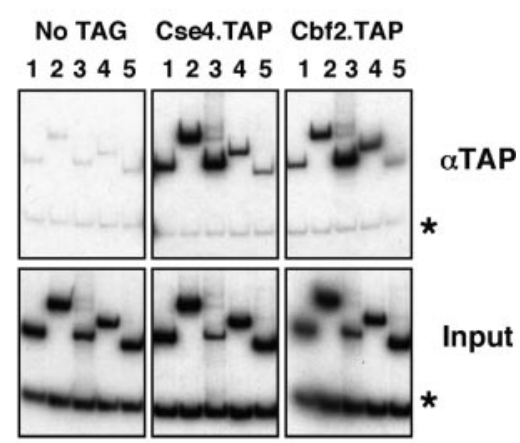

C

Htz1.TAP

H2A.TAP
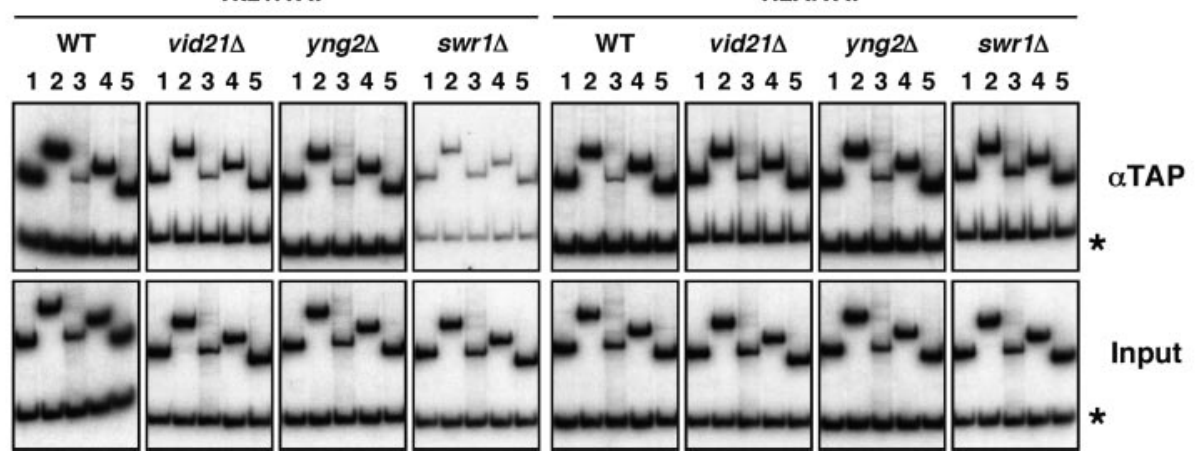

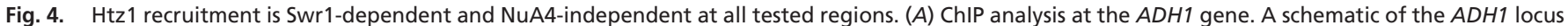

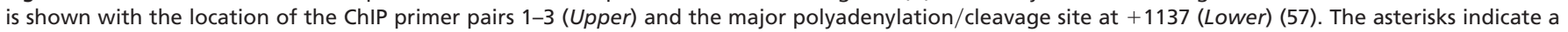
subtelomeric region of chromosome V (9716-9823) that we frequently use as a nontranscribed control when analyzing transcriptionally active regions (5).

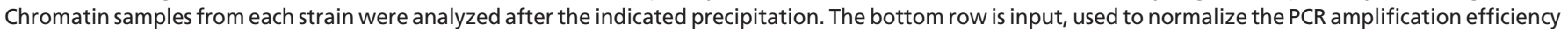

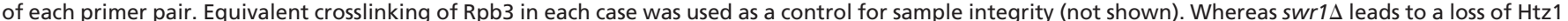

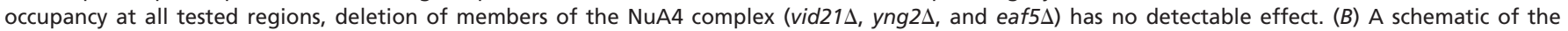

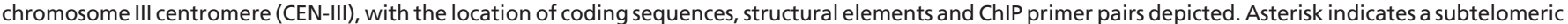

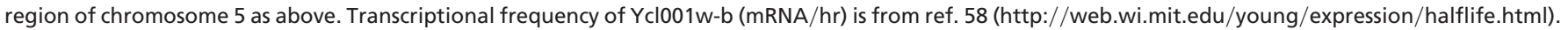

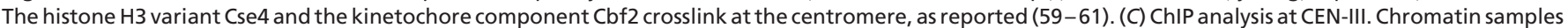

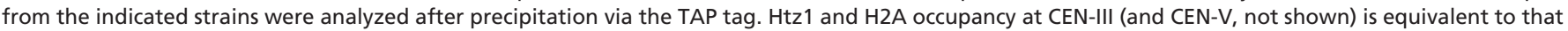

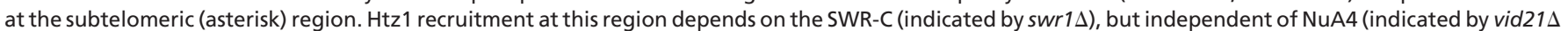

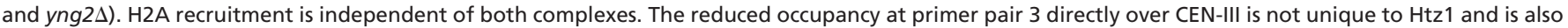

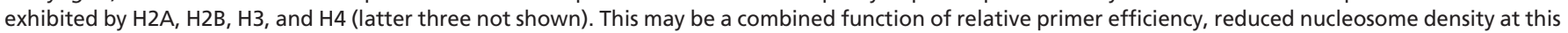
region, and steric occlusion by the kinetochore complex.

ically with the histone H3 K4 methylation pathway (Fig. 2A), deletions of genes encoding subunits of the PAF complex or COMPASS do not cause benomyl sensitivity (ref. 49 and our unpublished data), nor does deletion of RTF1, which encodes a subunit of the PAF complex, cause chromosome loss (50).

To further characterize the roles of the NuA4, the SWR-C, and Htz1 in chromosome stability, we quantified chromosome missegregation in vid $21 \Delta$, eaf $7 \Delta, y a f 9 \Delta, s w r 1 \Delta$, and $h t z 1 \Delta$ diploid strains by using colony half-sector analysis (24). In each case, we observed a significant increase in chromosome loss rates compared to wildtype diploids (Table 1). The role of Htz1 in this process has been conserved across species, as deletion of the H2A.Z variant, Pht1, from the fission yeast Schizosaccharomyces pombe is also associated with decreased chromosome stability (51), and mammalian H2A.Z plays a role in chromosome segregation (52). Although the eaf7 $\Delta$ strain displayed elevated chromosome instability rates, it was beno- 
myl insensitive, indicating that the two phenotypes are not fully correlated (as is also the case for certain kinetochore mutants; our unpublished data). The benomyl sensitivity and chromosome loss defects of a subset of NuA4 mutants, swr $1 \Delta$ and $h t z 1 \Delta$, suggest that the two complexes have functionally linked roles in chromosome segregation.

Because of the role of Htz1 in regulating gene expression $(5,9)$, its effect on chromosomal segregation could, in principle, be indirect. However, our microarray analyses using strains deleted for HTZ1 or components of the SWR-C did not detect any significant changes in the transcription of genes encoding kinetochore proteins (see www.utoronto.ca/greenblattlab/nua4swrc.xls). To ascertain whether Htz1 has a direct role in chromosome segregation, TAPtagged versions of Htz1 were analyzed by ChIP after proteins were cross-linked in vivo to DNA by using formaldehyde. Yeast cells were lysed and, after isolation and shearing of chromatin, the presence of Htz1 near specific DNA sequences was monitored by immunoprecipitating (IP) with IgG that binds to the protein A component of the TAP tag. After reversal of the cross-links, the coprecipitated DNA was analyzed by PCR amplification with specific primer pairs directed against regions of interest. Each PCR also contained primers directed against a nontranscribed subtelomeric region of chromosome 5, as we have previously determined that Htz1 occupancy is high at this location (5). In each case, we also examined the DNA that coprecipitated with the major histone H2A as a control.

As previously observed (5), Htz1 recruitment at the constitutively transcribed genes $A D H 1$ and $P M A 1$, a Htz1-dependent gene cluster (Htz1 activated domain), and the telomere of chromosome $\mathrm{V}$ required Swr1 (Fig. $4 A$ and data not shown). We now show that Htz1 is also recruited to centromeric regions in a Swr1-dependent manner (Fig. $4 C$ ), indicating that the SWR-C and Htz1 may directly regulate centromere function as well as transcription. In contrast, recruitment of the major histone $\mathrm{H} 2 \mathrm{~A}$ is independent of Swr1 at all of these locations (Fig. $4 A$ and $C$ ).

Despite the involvement of NuA4 in Htz1 function suggested by our genetic and microarray experiments, Htz1 recruitment at all

1. Lusser, A. \& Kadonaga, J. T. (2003) BioEssays 25, 1192-1200.

2. Strahl, B. D. \& Allis, C. D. (2000) Nature 403, 41-45.

3. Jenuwein, T. \& Allis, C. D. (2001) Science 293, 1074-1080.

4. Malik, H. S. \& Henikoff, S. (2003) Nat. Struct. Biol. 10, 882-891.

5. Krogan, N. J., Keogh, M.-C., Datta, N., Sawa, C., Ryan, O. W., Ding, H., Haw, R. A., Pootoolal, J., Tong, A., Canadien, V., et al. (2003) Mol. Cell 12, 1565-1576.

6. Mizuguchi, G., Shwn, X., Landry, J., Hu, W.-H., Sen, S. \& Wu, C. (2004) Science 303, 343-348.

7. Kobor, M. S., Venkatasubrahmanyam, S., Meneghini, M. D., Gin, J. W., Jennings, J. L., Link, A. J., Madhani, H. D. \& Rine, J. (2004) Pub. Lib. Sci. Biol. 2, 587-599.

8. Jackson, J. D. \& Gorovsky, M. A. (2000) Nucleic Acids Res. 28, 3811-3816.

9. Meneghini, M. D., Wu, M. \& Madhani, H. D. (2003) Cell 112, 725-736.

9. Meneghini, M. D., Wu, M. \& Madhani, H. D. (2003) Cell 112, 725-736.
10. Santisteban, M. S., Kalashnikova, T. \& Smith, M. M. (2000) Cell 103, 411-422.

11. Eberharter, A. \& Becker, P. B. (2002) EMBO Rep. 3, 224-229.

12. Turner, B. M. (1993) Cell 75, 5-8.

13. Matangkasombut, O. \& Buratowski, S. (2003) Mol. Cell 11, 353-363.

14. Doyon, Y. \& Cote, J. (2004) Curr. Opin. Gen. Dev. 14, 147-154.

15. Smith, E. R., Eisen, A., Gu, W., Sattah, M., Pannuti, A., Zhou, J., Cook, R. G., Lucchesi, J. C. \& Allis, C. D. (1998) Proc. Natl. Acad. Sci. USA 95, 3561-3565.

16. Allard, S., Utley, R. T., Savard, J., Clarke, A., Grant, P., Brandl, C. J., Pillus, L., Workman, J. L. \& Cote, J. (1999) EMBO J. 18, 5103-5119.

17. Gavin, A. C., Bosche, M., Krause, R., Grandi, P., Marzioch, M., Bauer, A., Schultz, J., Rick, J. M., Michon, A. M., Cruciat, C. M., et al. (2002) Nature 415, 141-147.

18. Krogan, N. J., Kim, M., Ahn, S. H., Zhong, G., Kobor, M. S., Cagney, G., Emili, A., Shilatifard, A., Buratowski, S. \& Greenblatt, J. F. (2002) Mol. Cell Biol. 22, 6979-6992.

19. Tong, A. H. Y., Evangalista, M., Parsons, A. B., Xu, H., Bader, G. D., Page, N., Robinson, M., Raghibizadeh, S., Hogue, C. W. V., Bussey, H., et al. (2001) Science 294, 2364-2368.

20. Hughes, T. R., Marton, M. J., Jones, A. R., Roberts, C. J., Stoughton, R., Armour, C. D. Bennett, H. A., Coffey, E., Dai, H., He, Y. D., et al. (2000) Cell 102, 109-126.

21. Peng, W. T., Robinson, M. D., Mnaimneh, S., Krogan, N. J., Cagney, G., Morris, Q., Davierwala, A. P., Grigull, J., Yang, X., Zhang, W., et al. (2003) Cell 113, 919-933.

22. Clarke, A. S. Lowell, J. E., Jacobson, S. J. \& Pillus, L. (1999) Mol. Cell. Biol. 19, 2515-2526

23. Le Masson, I., Yu, D. Y., Jensen, K., Chevalier, A., Courbeyrette, R., Boulard, Y., Smith, M. M. \& Mann, C. (2003) Mol. Cell. Biol. 23, 6086-6102.

24. Koshland, D. \& Hieter, P. (1987) Methods Enzymol. 155, 351-372.

25. Hyland, K., Kingsbury, J., Koshland, D. \& Hieter, P. (1999) J. Cell Biol. 145, 15-28.

26. Baetz, K. K., Krogan, N. J., Emili, A., Greenblatt, J. F. \& Hieter, P. (2004) Mol. Cell. Biol. 24, 1232-1244.

27. Ikura, T., Ogryzko, V. V., Grigoriev, M., Groisman, R., Wang, J., Horikoshi, M., Scully, R. Qin, J. \& Nakatani, Y. (2000) Cell 102, 463-473.

28. Cai, Y., Jin, L., Tomomori-Sato, C., Sato, S., Sorokina, I., Parmely, T. J., Conaway, R. C. \& Conaway, J. W. (2003) J. Biol. Chem. 278, 42733-42736.

29. Doyon, Y., Selleck, W., Lane, W. S., Tan, S. \& Cote, J. (2004) Mol. Cell. Biol. 24, 1884-1896. tested regions was independent of Vid21 and Yng2 (Fig. $4 A$ and $C$ and data not shown), suggesting that Htz1 recruitment is NuA4independent. We have also determined that Htz1 or H2A recruitment at all tested locations is unaffected after inactivation of temperature-sensitive alleles of esa1 (esa1- $\Delta 414$ and esa1-L327S) (data not shown). It seems likely, therefore, that $\mathrm{NuA} 4$ has a postrecruitment role, as yet undefined, in Htz1 function. Because $\mathrm{NuA} 4$ is known to acetylate histone $\mathrm{H} 2 \mathrm{~A}$, it is tempting to speculate that its role in the SWR-C/Htz1 pathway is to acetylate the histone $\mathrm{H} 2 \mathrm{~A}$ variant Htz1 after its incorporation into chromatin. H2A.Z $\mathrm{N}$-terminal tail acetylation is essential in Tetrahymena $(53,54)$, and covalent modification may be required for at least some of the functions performed by this histone variant in $S$. cerevisiae.

We have shown here that the NuA4 HAT complex has three previously unidentified subunits and that the Vid21 and Yng2 subunits of $\mathrm{NuA} 4$ have synthetic genetic interactions and effects on gene expression very similar to those of Htz1 and the SWR-C. We have also found that Htz1 is recruited to centromeric regions and mutations in the genes encoding Htz1, the SWR-C, and subunits of $\mathrm{NuA} 4$ lead to a decrease in the fidelity of chromosome segregation. Htz1 may contribute to the overall structure of centromeric chromatin in such a way as to affect centromere function and chromosome transmission (55). Because the HAT activity of NuA4 on histone $\mathrm{H} 4$ is unnecessary for recruiting Htz1 into chromatin, we predict that NuA4 directly acetylates Htz1 to influence its function.

We thank Lorraine Pillus (University of California at San Diego, La Jolla) for strain LPY3500, Jacques Cote for sharing unpublished data, and Affinium Pharmaceuticals for help with MALDI-TOF mass spectrometry. N.J.K. was supported by a Doctoral Fellowship from the Canadian Institutes of Health Research (CIHR), and K.B. was supported by Postdoctoral Fellowships from CIHR and the Michael Smith Foundation for Health Research. This research was supported by grants from the CIHR, the Ontario Genomics Institute, and the National Cancer Institute of Canada with funds from the Canadian Cancer Society (to J.F.G.), and National Institutes of Health Grants GM46498 (to S.B.) and CA16591 (to P.H.). S.B. is a Scholar of the Leukemia and Lymphoma Society.

30. Gadal, O., Labarre, S., Boschiero, C. \& Thuriaux, P. (2002) EMBO J. 21, 5498-5507.

31. Reid, J. L., Iyer, V. R., Brown, P. O. \& Struhl, K. (2000) Mol. Cell 6, 1297-1307.

32. Hampsey, M. \& Reinberg, D. (2003) Cell 113, 429-432.

33. Krogan, N. J., Dover, J., Wood, A., Schneider, J., Heidt, J., Boateng, M. A., Dean, K., Ryan, O. W., Golshani, A., Johnston, M., et al. (2003) Mol. Cell 11, 721-729.

34. Ng, H. H., Robert, F., Young, R. A. \& Struhl, K. (2003) Mol. Cell 11, 709-719.

35. Dover, J., Schneider, J., Tawiah-Boateng, M. A., Wood, A., Dean, K., Johnston, M. \& Shilatifard, A. (2002) J. Biol. Chem. 277, 28368-28371.

36. Sun, Z. W. \& Allis, C. D. (2002) Nature 418, 104-108.

37. Santos-Rosa, H., Schneider, R., Bernstein, B. E., Karabetsou, N., Morillon, A., Weise, C. Schreiber, S. L., Mellor, J. \& Kouzarides, T. (2003) Mol. Cell 12, 1325-1332.

38. Choy, J. S., Tobe, B. T., Huh, J. H. \& Kron, S. J. (2001) J. Biol. Chem. 276, 43653-43662.

39. Ruiz-Garcia, A. B., Sendra, R., Galiana, M., Pamblanco, M., Perez-Ortin, J. E. \& Tordera, V. (1998) J. Biol. Chem. 273, 12599-12605.

40. Mcainsh, A. D., Tytell, J. D. \& Sorger, P. K. (2003) Annu. Rev. Cell Dev. Biol. 19, 519-539. 41. Measday, V. \& Hieter, P. (2004) Nat. Cell Biol. 6, 94-95.

42. Stoler, S., Keith, K. C., Curnick, K. E. \& Fitzgerald-Hayes, M. (1995) Genes Dev. 9, 573-586

43. Strunnikov, A. V., Kingsbury, J. \& Koshland, D. (1995) J. Cell Biol. 128, 749-760.

44. Doheny, K. V., Sorger, P. K., Hyman, A. A., Tugenderich, S., Spencer, F. \& Hieter, P. (1993) Cell 73, 761-764.

45. Ortiz, J., Stemmann, O., Rank, S. \& Lechner, J. (1999) Genes Dev. 13, 1140-1155.

46. Gillett, E. S., Espelin, C. W. \& Sorger, P. K. (2004) J. Cell Biol. 164, 535-546.

47. Krishnan, R., Pangilinian, F., Lee, C. \& Spencer, F. (2000) Genetics 156, 489-500.

48. Fleming, J. A., Vega, L. R. \& Solomon, F. (2000) Genetics 156, 69-80.

49. Betz, J. L., Chang, M., Washburn, T. M., Porter, S. E., Mueller, C. L. \& Jaehning, J. A. (2002) Mol. Genet. Genomics 268, 272-285.

50. Baker, R. E., Harris, K. \& Zhang, K. (1998) Genetics 149, 73-85.

51. Carr, A. M., Dorrington, S. M., Hindley, J., Phear, G. A., Aves, S. J. \& Nurse, P. (1994) Mol. Gen. Genet. 245, 628-635.

52. Rangasamy, D., Greaves, I. \& Tremethick, D. J. (2004) Nat. Struct. Mol. Biol. 11, 650-655. 53. Ren, Q. \& Gorovsky, M. A. (2001) Mol. Cell 7, 1329-1335.

54. Ren, Q. \& Gorovsky, M. A. (2003) Mol. Cell. Biol. 23, 2778-2789.

55. Sharp, J. A. \& Kaufman, P. D. (2003) Curr. Top. Microbiol. Immunol. 274, 23-52

56. Enenkel, C., Blobel, G. \& Rexach, M. (1995) J. Biol. Chem. 270, 16499-16502.

57. Kim, M., Ahn, S. H., Krogan, N. J., Greenblatt, J. F. \& Buratowski, S. (2004) EMBO J. 23, 354-364.

58. Holstege, F. C., Jennings, E. G., Wyrick, J. J., Lee, T. I., Hengartner, C. J., Green, M. R Golub, T. R., Lander, E. S. \& Young, R. A. (1998) Cell 95, 717-728.

59. Meluh, P. B., Yang, P., Glowczewski, L., Koshland, D. \& Smith, M. M. (1998) Cell 94, 607-613.

60. Jiang, W., Lechner, J. \& Carbon, J. (1993) J. Cell Biol. 121, 513-519.

61. Meluh, P. B. \& Koshland, D. (1997) Genes Dev. 11, 3410-3412. 